\title{
Relativistic Electron Vortices
}

\author{
Stephen M. Barnett \\ School of Physics and Astronomy, University of Glasgow, Glasgow G12 8QQ, United Kingdom
}

(Received 25 November 2016; published 13 March 2017)

\begin{abstract}
The desire to push recent experiments on electron vortices to higher energies leads to some theoretical difficulties. In particular the simple and very successful picture of phase vortices of vortex charge $\ell$ associated with $\ell \hbar$ units of orbital angular momentum per electron is challenged by the facts that (i) the spin and orbital angular momentum are not separately conserved for a Dirac electron, which suggests that the existence of a spin-orbit coupling will complicate matters, and (ii) that the velocity of a Dirac electron is not simply the gradient of a phase as it is in the Schrödinger theory suggesting that, perhaps, electron vortices might not exist at a fundamental level. We resolve these difficulties by showing that electron vortices do indeed exist in the relativistic theory and show that the charge of such a vortex is simply related to a conserved orbital part of the total angular momentum, closely related to the familiar situation for the orbital angular momentum of a photon.
\end{abstract}

DOI: 10.1103/PhysRevLett.118.114802

Vortices are ubiquitous wherever we have a fluid in motion. They have been observed and studied in many settings including in classical [1,2] and quantum fluids [3-5], in nonlinear fields [6], and also in optics [7], where their presence is associated with an orbital angular momentum [8,9]. Recent developments have demonstrated, beyond reasonable doubt, the existence of propagating electrons with an on-axis vortex, corresponding to a phase singularity in the wave function of the form $e^{i \ell \phi}$, where $\phi$ is the azimuthal coordinate [10-19]. Comparison with paraxial optics [20,21] or direct appeal to quantum theory [22,23] leads us to associate these with $\ell \hbar$ units of orbital angular about the direction of propagation. The experimental studies of electron vortices are backed by an impressive array of theoretical developments [24-32] and it may be said justifiably that the phenomena of electron vortices and the associated electron orbital angular momentum are now well understood.

Theoretical investigations at higher energies, based on the Dirac equation, have given cause to question the above simple statements. As is well known, the spin and orbital parts of the electron angular momentum are not separately conserved [33] and this suggests the existence of a spinorbit coupling rather than a simple orbital angular momentum associated with a vortex beam [34]. The velocity of a Dirac electron, moreover, is not proportional to the momentum operator [35] and hence the local velocity is not the gradient of a phase [36]. These two features give cause to question the validity of our simple nonrelativistic

Published by the American Physical Society under the terms of the Creative Commons Attribution 4.0 International license. Further distribution of this work must maintain attribution to the author(s) and the published article's title, journal citation, and DOI. analysis, with its phase vortices associated with an orbital angular momentum. Even the existence of the vortices seems to be in doubt [36].

There are three reasons why this problem is important: one practical and two fundamental. The first derives from the requirement to be able to describe, as simply as possible, experiments with shaped electron beams as they move towards higher energies. The second is the question of whether electron vortices are real and if they are, as experiments suggest, what happens to these topological features in the relativistic limit? Finally, we have long associated phase vortices with a well-defined orbital angular momentum, but Dirac theory tells us that the orbital and spin parts of the angular momentum are not separately conserved. Is this at odds, however, with the existence of electron vortices? We are encouraged by the fact that analogous issues arose and were resolved for light, which is the quintessential relativistic field. To address these points we ask if there is any sense in which the simplicity of the nonrelativistic description of electron vortices persists in the relativistic domain. We find that there is.

We work in the natural system of units in which $\hbar=1=c$ and write our Dirac equation in the form

$$
i \frac{\partial \psi}{\partial t}=(\boldsymbol{\alpha} \cdot \mathbf{p}+\beta m) \psi
$$

where $\psi$ is the usual four-component spinor and $\alpha$ and $\beta$ are the matrices [35]

$$
\boldsymbol{\alpha}=\left(\begin{array}{cc}
0 & \boldsymbol{\sigma} \\
\boldsymbol{\sigma} & 0
\end{array}\right), \quad \beta=\left(\begin{array}{cc}
\mathrm{I} & 0 \\
0 & -\mathrm{I}
\end{array}\right) .
$$

The orbital and spin angular momentum operators take the expected forms 


$$
\mathbf{L}=\mathbf{x} \times \mathbf{p}, \quad \mathbf{S}=\frac{1}{2}\left(\begin{array}{cc}
\boldsymbol{\sigma} & 0 \\
0 & \boldsymbol{\sigma}
\end{array}\right),
$$

but note that neither $\mathbf{L}$ nor $\mathbf{S}$ alone commutes with the Dirac Hamiltonian $H=\boldsymbol{\alpha} \cdot \mathbf{p}+\beta m$. Only the total angular momentum $\mathbf{J}=\mathbf{L}+\mathbf{S}$ commutes with $H$ and is conserved [33]. This situation contrasts strongly with the nonrelativistic Hamiltonian for the free electron

$$
H_{\text {nonrel }}=\frac{p^{2}}{2 m},
$$

which manifestly commutes with both $\mathbf{L}$ and $\mathbf{S}$ so that each of these is separately conserved. It is straightforward, moreover, to find propagating-electron solutions of the Schrödinger equation with azimuthal dependence $e^{i \ell \phi}$, which are eigenstates of $L_{z}$ with eigenvalue $\ell$ [26].

In seeking to link the relativistic and nonrelativistic treatments it is natural to employ the Foldy-Wouthuysen transformation as it is the low energy limit of this form that leads to the Schrödinger equation [35,37]. To this end we introduce the unitary operator

$$
e^{i S}=e^{\beta \boldsymbol{\alpha} \cdot \mathbf{p} \theta / p}, \quad \tan (2 \theta)=\frac{p}{m}
$$

to transform our spinor to $\psi^{\prime}=e^{i S} \psi$. This transformation diagonalizes the Dirac Hamiltonian

$$
H^{\prime}=e^{i S} H e^{-i S}=\beta\left(p^{2}+m^{2}\right)^{1 / 2},
$$

so that our transformed Dirac equation is

$$
i \frac{\partial \psi^{\prime}}{\partial t}=\beta\left(p^{2}+m^{2}\right)^{1 / 2} \psi^{\prime} .
$$

It should be emphasized that this is an exact form of the Dirac equation for the free electron and that no approximation has been made in order to obtain it.

The transformed Dirac Hamiltonian $H^{\prime}$ commutes with both $\mathbf{L}$ and $\mathbf{S}$ and it follows that, in the transformed picture, these quantities are separately conserved. The unitary operator $e^{i S}$ commutes with $\mathbf{J}$, moreover, and the above observation provides the basis for extracting two separately conserved parts of the angular momentum.

We can write exact solutions of our transformed Dirac equation (7) with an aximuthal dependence $e^{i \ell \phi}$ and these will have a phase vortex along the $z$ axis. These solutions will be eigenstates, moreover, of the operator $L_{z}=-i \partial / \partial \phi$. Rather than work with the exact Dirac equation, it is helpful to introduce an approximation at this stage, so as to make a natural link with previous studies and with experiments. To this end let us assume that the electron motion is principally in the $z$ direction, by which we mean that $p_{z} \gg p_{x}, p_{y}$. We further specialize, for simplicity, to a monoenergetic state with energy $\left(m^{2}+p_{0}^{2}\right)^{1 / 2}$. This restricts us, naturally, to the twocomponent upper part of our spinor $\psi^{\prime}$. Finally, let us write

$$
\psi_{\text {upper }}^{\prime}=e^{-i \sqrt{m^{2}+p_{0}^{2}} t} e^{i p_{0} z} u(\mathbf{r})\left(\begin{array}{l}
a \\
b
\end{array}\right),
$$

where $a$ and $b$ determine the orientation of the electron spin and $|a|^{2}+|b|^{2}=1$. If we expand the Hamiltonian operator

$$
\left(m^{2}+p^{2}\right)^{1 / 2} \approx \sqrt{m^{2}+p_{0}^{2}}+\frac{p_{x}^{2}+p_{y}^{2}}{2 \sqrt{m^{2}+p_{0}^{2}}}+\frac{p_{0}\left(p_{z}-p_{0}\right)}{\sqrt{m^{2}+p_{0}^{2}}}
$$

then we are led to

$$
i \frac{\partial u}{\partial z}=-\frac{1}{2 p_{0}}\left(\frac{\partial^{2}}{\partial x^{2}}+\frac{\partial^{2}}{\partial y^{2}}\right) u,
$$

which is the paraxial wave equation familiar from optics [38]. This is entirely natural as we can write Maxwell's equations for the free electromagnetic field in Dirac form and applying to these the appropriate Foldy-Wouthuysen transformation and making the paraxial approximation leads to the same equation [39]. Note that in deriving this equation we have placed no restriction on the value of $p_{0}$ and that, despite its similarity to the Schrödinger equation, it is a fully relativistic expression. Solutions to this equation include the Laguerre-Gaussian modes, familiar from optics $[20,21]$, with the desired azimuthal dependence $e^{i \ell \phi}$.

We have solutions for $\psi^{\prime}$ that are eigenstates of the $z$ component of the orbital angular momentum operator, $L_{z}=x p_{y}-y p_{x}$, with integer eigenvalue $\ell$. We can also select eigenstates of the $z$ component of the spin, $S_{z}$, with eigenvalue $\pm 1 / 2$ by choosing $a=1$ or $b=1$ in our solution (8). It remains to determine the forms of these observables for the original Dirac equation. Clearly, they will not simply be $L_{z}$ and $S_{z}$ as these operators do not commute with the unitary operator $e^{i S}$. We find in this way two separately conserved parts of the total angular momentum for a free Dirac electron:

$$
\begin{aligned}
\tilde{\mathbf{L}}= & e^{-i S} \mathbf{x} \times \mathbf{p} e^{i S}=\mathbf{x} \times \mathbf{p}+i \frac{\beta \boldsymbol{\alpha} \times \mathbf{p}}{\sqrt{m^{2}+p^{2}}} \\
& +\left(1-\frac{m}{\sqrt{m^{2}+p^{2}}}\right)\left(\mathbf{S}-\frac{(\mathbf{p} \cdot \mathbf{S}) \mathbf{p}}{p^{2}}\right), \\
\tilde{\mathbf{S}}= & e^{-i S} \mathbf{S} e^{i S}=\mathbf{S}-i \frac{\beta \boldsymbol{\alpha} \times \mathbf{p}}{\sqrt{m^{2}+p^{2}}} \\
& -\left(1-\frac{m}{\sqrt{m^{2}+p^{2}}}\right)\left(\mathbf{S}-\frac{(\mathbf{p} \cdot \mathbf{S}) \mathbf{p}}{p^{2}}\right) .
\end{aligned}
$$


The physical significance of these remains to be fully established. Each, however, is a separately conserved part of the total angular momentum for a free Dirac electron and the $z$ components, in particular, correspond to the conserved quantities $L_{z}$ and $S_{z}$ for our solution to the transformed Dirac equation, $\psi^{\prime}$. It follows that for a transformed state $\psi^{\prime}$ with an azimuthal dependence $e^{i \ell \phi}$ the corresponding state $\psi$ will be an eigenstate of $\tilde{L}_{z}$ with eigenvalue $\ell$.

We may ask how to interpret and even name the separately conserved parts of the total angular momentum. It is clear, by their construction, that $\tilde{\mathbf{L}}$ and $\tilde{\mathbf{S}}$ are associated with phase vortices and the electron spin that appear in the transformed state $\psi^{\prime}$. For this reason, if no other, it seems appropriate to call $\tilde{\mathbf{L}}$ the conserved orbital part of the electron angular momentum and $\tilde{\mathbf{S}}$, correspondingly, the conserved spin part [40]. The $z$ component of the conserved orbital part of the angular momentum, $\tilde{L}_{z}$, is clearly associated with the presence of a phase vortex in $\psi^{\prime}$ such as occurs on the $z$ axis for a Laguerre-Gaussian mode.

It should again be emphasized that Eq. (7) is an exact reexpression of the Dirac equation and that we can construct any positive energy solution by superposing plane waves with components in the upper half of the spinor. For practical purposes, however, the paraxial approximate form will often suffice, just as it does in optics. Let us construct a solution with an on-axis vortex associated with the conserved orbital part of the angular momentum. Direct comparison with the paraxial modes from optics $[20,21,38]$ leads us to write [44]

$$
\begin{aligned}
u_{\ell, n}= & \sqrt{\frac{2 n !}{\pi(n+|\ell|) !}} \frac{(\rho \sqrt{2})^{|\ell|}}{w^{|\ell|+1}(z)} \exp \left(-\frac{p_{0} \rho^{2}}{2\left(z_{R}+i z\right)}\right) \\
& \times L_{n}^{|\ell|}\left(\frac{2 \rho^{2}}{w^{2}(z)}\right) e^{i \ell \phi} \exp \left[-i(2 n+|\ell|+1) \tan ^{-1}\left(z / z_{R}\right)\right],
\end{aligned}
$$

where we have used cylindrical polar coordinates, $w(z)=w(0) / \sqrt{1+z^{2} / z_{R}^{2}}$, and $z_{R}$ is the Rayleigh range $p_{0} w^{2}(0) / 2$. The full solution for $\psi^{\prime}$ is

$$
\psi^{\prime}=e^{-i \sqrt{m^{2}+p_{0}^{2}} t} e^{i p_{0} z} u_{\ell, n}\left(\begin{array}{l}
a \\
b \\
0 \\
0
\end{array}\right) .
$$

If we wish to work with the Dirac equation in its original form then this solution becomes

$$
\psi=e^{-i S} \psi^{\prime},
$$

which will have nonzero entries in both the upper and lower parts of the spinor, corresponding to a superposition of positive and negative energy states of the stationary electron.

We have established the existence of vortex solutions and, within the paraxial approximation, Laguerre-Gaussian solutions akin to those arising in optics, but these are for the transformed state $\psi^{\prime}$ rather than $\psi$ and it remains to show that vortices appear also for $\psi$. This point is far from obvious, as the position coordinates in the two states are not the same by virtue of the fact that $e^{-i S}$ depends on the momentum operator. The coordinate transformation mixes positions over a distance of the order of the Compton wavelength for the electron and it suffices, therefore, to consider only the region of space that is very close to the $z$ axis. We further simplify the analysis by considering the focal plane $z=0$ and hence write

$$
\psi^{\prime} \approx N e^{-i \sqrt{m^{2}+p_{0}^{2}} t} e^{i p_{0} z}(x+i y)^{\ell}\left(\begin{array}{l}
a \\
b \\
0 \\
0
\end{array}\right),
$$

where $N$ is a normalization constant and we have chosen $\ell$ to be positive. It is straightforward to perform the required unitary transformation and we find

$$
\begin{aligned}
\psi= & \frac{N}{\sqrt{2}} e^{-i \sqrt{m^{2}+p_{0}^{2}} t} e^{i p_{0} z}\left[u_{+}(x+i y)^{\ell}\left(\begin{array}{l}
a \\
b \\
0 \\
0
\end{array}\right)\right. \\
& \left.+u_{-}\left((x+i y)^{\ell}\left(\begin{array}{c}
0 \\
0 \\
a \\
-b
\end{array}\right)-i \frac{2 \ell}{p_{0}}(x+i y)^{\ell-1}\left(\begin{array}{l}
0 \\
0 \\
b \\
0
\end{array}\right)\right)\right],
\end{aligned}
$$

where $u_{ \pm}=\left(1 \pm m / \sqrt{m^{2}+p_{0}^{2}}\right)^{1 / 2}$. That the phase vortex behavior remains is clear from the presence of the terms $(x+i y)^{\ell}=\rho^{\ell} e^{i \ell \phi}$. Some quenching of the vortex occurs if the electron is spinning in the opposite sense to the rotation of the vortex in that very near the center the state with a vortex charge reduced to $\ell-1$ and the electron spinning in the opposite direction will dominate. The length scale for this to happen is very small, however, being of the order of a few Compton wavelengths. We see that phase vortex solutions for the transformed state $\psi^{\prime}$ occur very naturally and, moreover, that these persist when transformed back into the original form of the Dirac state.

The final question to address is whether or not there is an azimuthal current flow associated with the region around the vortex. The point is that the electron velocity operator is $\boldsymbol{\alpha}$ and that this leads to a local current that is not the gradient 
of the phase of the spinor [36]. To address this point we evaluate, within our paraxial approximation, the expectation value of the $z$ component of $\mathbf{x} \times \boldsymbol{\alpha}$ for our solution (13) [45]:

$$
\begin{aligned}
\left\langle(\mathbf{x} \times \boldsymbol{\alpha})_{z}\right\rangle & =\int d^{3} x \psi^{\prime \dagger} e^{i S}\left(x \alpha_{y}-y \alpha_{x}\right) e^{-i S} \psi^{\prime} \\
& =\int d^{3} x \frac{1}{\sqrt{m^{2}+p_{0}^{2}}} \psi^{\prime \dagger}\left(-i \frac{\partial}{\partial \phi}\right) \psi^{\prime} \\
& =\frac{\ell}{\sqrt{m^{2}+p_{0}^{2}}} .
\end{aligned}
$$

It follows that we have a net azimuthal current around the vortex. Moreover, as this quantity gives the expectation value of the cross product of the position and the velocity, we can think of the product of this quantity and the energy $\sqrt{m^{2}+p_{0}^{2}}$ as being the orbital angular momentum, although strictly speaking it is the expectation value of $\tilde{L}_{z}$.

It remains to assess our progress in addressing the three important issues that motivated this study and were listed at the beginning of this Letter. First, we have shown that the same paraxial methods of solution that have been used in optics and for low energy electrons can be applied in the fully relativistic regime. This means that much of the simplicity of the low energy solutions is retained. We have shown also that electron vortices are indeed real in the relativistic limit, but that the natural way to look for them is to work with the Foldy-Wouthuysen transformed spinor. Finally, the electron vortex is indeed associated with a welldefined and conserved part of the total angular momentum, but that this quantity is $\tilde{\mathbf{L}}$, given in Eq. (11), rather than the simple orbital angular momentum we find for low energies.

We conclude by noting that the description given here applies for the free electron, but that simple electron vortex solutions have been found, also, for propagation in a constant magnetic field pointing along the direction of propagation [25]. Extending our relativistic analysis to this situation presents no fundamental difficulties; we can introduce a vector potential with components in the $x-y$ plane and then apply a two-stage Foldy-Wouthuysen transformation [35], treating the transverse momentum components as small. This leads to a paraxial wave equation similar in form to Eq. (11) but with $-i \partial / \partial x$ $(-i \partial / \partial y)$ replaced by $p_{x}-e A_{x}\left(p_{y}-e A_{y}\right)$ and a Zeeman energy shift associated with the electron spinning in the magnetic field. The ideas expressed here are not restricted to electrons but apply also to relativistic motion of other spin-half particles including neutrons, for which the existence of phase vortices has recently been demonstrated [46].

I thank Iwo Bialynicki-Birula for kindly sending me a preprint of Ref. [36]. This work was supported by a Royal Society Research Professorship Grant No. RP150122.
[1] P. G. Saffman, Vortex Dynamics (Cambridge University Press, Cambridge, 1992).

[2] A. J. Majda and A. L. Bertozzi, Vorticity and Incompressible Flow (Cambridge University Press, Cambridge, 2002).

[3] D. R. Tilley and J. Tilley, Superfluidity and Superconductivity (Institute of Physics Publishing, Bristol, 1990).

[4] R. J. Donnelly, Quantized Vortices in Helium II (Cambridge University Press, Cambridge, England, 1991).

[5] A. J. Leggett, Quantum Liquids (Oxford University Press, Oxford, 2006).

[6] L. M. Pismen, Vortices in Nonlinear Fields (Oxford University Press, Oxford, 1999).

[7] J. F. Nye, Natural Focussing and Fine Structure of Light (Institute of Physics Publishing, Bristol, 1999).

[8] A. M. Yao and M. J. Padgett, Adv. Opt. Photonics 3, 161 (2011).

[9] The Angular Momentum of Light, edited by D. L. Andrews and M. Babiker (Cambridge University Press, Cambridge, England, 2013).

[10] M. Uchida and A. Tonomura, Nature (London) 464, 737 (2010).

[11] J. Verbeeck, H. Tian, and P. Schattschneider, Nature (London) 467, 301 (2010).

[12] B. J. McMorran, A. Agarwal, I. M. Anderson, A. A. Herzing, H. J. Lezer, J. J. McClelland, and J. Unguris, Science 331, 192 (2011).

[13] P. Schattschneider, M. Stöger-Pollach, and J. Verbeeck, Phys. Rev. Lett. 109, 084801 (2012).

[14] G. Guzzinati, P. Schattschneider, K. Y. Bliokh, F. Nori, and J. Verbeeck, Phys. Rev. Lett. 110, 093601 (2013).

[15] K. Saitoh, Y. Hasegawa, K. Hirakawa, N. Tanaka, and M. Uchida, Phys. Rev. Lett. 111, 074801 (2013).

[16] G. Guzzinati, L. Clark, A. Béché, and J. Verbeeck, Phys. Rev. A 89, 025803 (2014).

[17] A. M. Blackburn and J. C. London, Ultramicroscopy 136, 127 (2014).

[18] R. Shiloh, Y. Tsur, R. Remez, Y. Lereah, B. A. Malomed, V. Shvedov, C. Hnatovsky, W. Krolikowski, and A. Arie, Phys. Rev. Lett. 114, 096102 (2015).

[19] V. Grillo, A. H. Tavabi, F. Venturi, H. Larocque, R. Balboni, G. C. Gazzadi, S. Frabboni, P.-H. Lu, E. Mafakheri, F. Bouchard, R. E. Durnin-Borkowski, R. W. Boyd, M. P. J. Lavery, M. J. Padgett, and E. Karimi, arXiv:1609.09129.

[20] L. Allen, M. W. Beijersbergen, R. J. C. Spreeuw, and J. P. Woerdman, Phys. Rev. A 45, 8185 (1992).

[21] L. Allen, S. M. Barnett, and M. J. Padgett, Optical Angular Momentum (Institute of Physics Publishing, Bristol, 2003).

[22] A. R. Edmonds, Angular Momentum in Quantum Mechanics (Princeton University Press, Princeton, 1957).

[23] C. Cohen-Tannoudji, B. Diu, and F. Laloë, Quantum Mechanics, Vol. 1 (Wiley, New York, 1977).

[24] I. Bialynicki-Birula, Z. Bialynicka-Birula, and C. Śliwa, Phys. Rev. A 61, 032110 (2000).

[25] K. Y. Bliokh, Y. P. Bliokh, S. Savel'ev, and F. Nori, Phys. Rev. Lett. 99, 190404 (2007).

[26] P. Schattschneider and J. Verbeeck, Ultramicroscopy 111, 1461 (2011).

[27] S. Lloyd, M. Babiker, and J. Yuan, Phys. Rev. Lett. 108, 074802 (2012). 
[28] S. M. Lloyd, M. Babiker, and J. Yuan, Phys. Rev. A 86, 023816 (2012).

[29] C. R. Greenshields, R. L. Stamps, and S. Franke-Arnold, New J. Phys. 14, 103040 (2012).

[30] K. Y. Bliokh, P. Schattschneider, J. Verbeeck, and F. Nori, Phys. Rev. X 2, 041011 (2012).

[31] C. R. Greenshields, R. L. Stamps, S. Franke-Arnold, and S. M. Barnett, Phys. Rev. Lett. 113, 240404 (2014).

[32] M. Babiker, J. Yuan, and V. E. Lembessis, Phys. Rev. A 91, 013806 (2015).

[33] P. A. M. Dirac, Proc. R. Soc. A 117, 610 (1928).

[34] K. Y. Bliokh, M. R. Dennis, and F. Nori, Phys. Rev. Lett. 107, 174802 (2011).

[35] J. D. Bjorken and S. D. Drell, Relativistic Quantum Mechanics (McGraw-Hill, New York, 1964).

[36] I. Bialynicki-Birula and Z. Bialynicka-Birula, preceding Letter, Phys. Rev. Lett. 118, 114801 (2017).

[37] L. L. Foldy and S. A. Wouthuysen, Phys. Rev. 78, 29 (1950).

[38] A. E. Siegman, Lasers (University Science Books, Sausalito, 1986).
[39] S. M. Barnett, New J. Phys. 16, 093008 (2014).

[40] This subtlety is reminiscent of the situation that occurs for light; we can identify conserved spin and orbital parts of the total angular momentum although neither of these, due to constraints of transversality, is a true angular momentum [41-43].

[41] S. J. van Enk and G. Nienhuis, J. Mod. Opt. 41, 963 (1994).

[42] S. J. van Enk and G. Nienhuis, Europhys. Lett. 25, 497 (1994).

[43] S. M. Barnett, J. Mod. Opt. 57, 1339 (2010).

[44] We have replaced the usual radial number $p$ by $n$ so as to avoid possible confusion with the momentum.

[45] Strictly speaking, to ensure a normalizable state, we should make $\psi^{\prime}$ a superposition over a small range of momenta $p_{0}$, but this can be an arbitrarily narrow distribution and so does not change our analysis in any essential way.

[46] C. W. Clark, R. Barankov, M. G. Huber, M. Arif, D. G. Cory, and D. A. Pushkin, Nature (London) 525, 504 (2015). 